\title{
USO CATAFORICO DEL TRAPASSATO PROSSIMO ITALIANO: UN ESPEDIENTE TESTUALE PER LA MESSA IN RILIEVO
}

\section{Introduzione}

Alcuni anni fa in un libro di testo per stranieri (Contesti italiani) mi sono imbattuta in un brano tratto dal romanzo di Giorgio Bassani Il giardino dei Finzi-Contini. Sebbene fosse costituito quasi per intero da Trapassati prossimi (brevemente TP) il commento pedagogico non sfiorava nemmeno l'argomento. Il materiale linguistico proposto non offriva una giustificazione sufficiente della scelta e, volendo scoprirla, ho cercato la risposta nel contesto dell'originale. Si trattava del capitolo 5 della prima parte, che inizia così:

\section{I.5}

Una volta, tuttavia, nel giugno del '29, il medesimo giorno in cui nell'atrio del Guarini ERANO STATE esposte le votazioni degli esami di licenza ginnasiale, ERA ACCADUTO qualcosa di molto più diretto e particolare.

Agli orali non ERO ANDATO granché bene.

Nonostante che il professor Meldolesi si fosse adoperato parecchio in mio favore, ottenendo addirittura, contro ogni regola, di essere lui stesso ad interrogarmi, quasi mai ERO APPARSO all'altezza dei numerosi sette e otto che ornavano la mia pagella nelle materie letterarie. (GFC-32)

Il brano assunto nei Contesti cominciava però solo dal secondo capoverso, cioè da Agli orali (...), e i Trapassati contenutivi - cominciando da non ero andato ed ero apparso - avevano sì trovato la loro immediata spiegazione nel legame tra le azioni a cui si riferivano e le due azioni designate nel capoverso ommesso. Ma il motivo poi per l'uso del nuovo Trapassato era accaduto l'ho trovato solo molto più tardi.

\section{Tipiche funzioni testuali del Trapassato prossimo}

Il TP è un paradigma verbale intrinsecamente anaforico, in quanto il suo significato di base presuppone un movimento all'indietro nel tempo partendo da un dato punto di riferimento. Si usa quindi per indicare vari tipi di anteriorità, così nella 
diegesi che nei discorsi riportati. Bassani ad es. lo usa in modo massiccio sia nei frequenti discorsi indiretti liberi, sia per i suoi altrettanto numerosi flash-back. Il punto di riferimento per i Trapassati nel brano citato (da non ero andato in poi) si trova quindi nella situazione menzionata in precedenza, e questa tecnica espositiva è sfruttata in non poche aperture di capitolo nel Giardino: l' autore ci svela l' avvenimento centrale (per lo più espresso in un Passato remoto (PR)), per poi tornare indietro nel tempo e presentare una serie di azioni che a questo evento cruciale avevano portato. Ecco uno di tali inizi (si veda anche l'illustrazione nel grafico No 1):

III.7

A casa nostra, quell' anno, la Pasqua venne celebrata con una cena sola. ERA STATO mio padre a volere così. Data anche l'assenza di Ernesto AVEVA DETTO - una Pasqua tipo quelle degli anni passati dovevamo scordarcela. E poi, a parte questo, in che modo avremmo potuto? (GFC-149)

\section{tempo del mondo testuale}

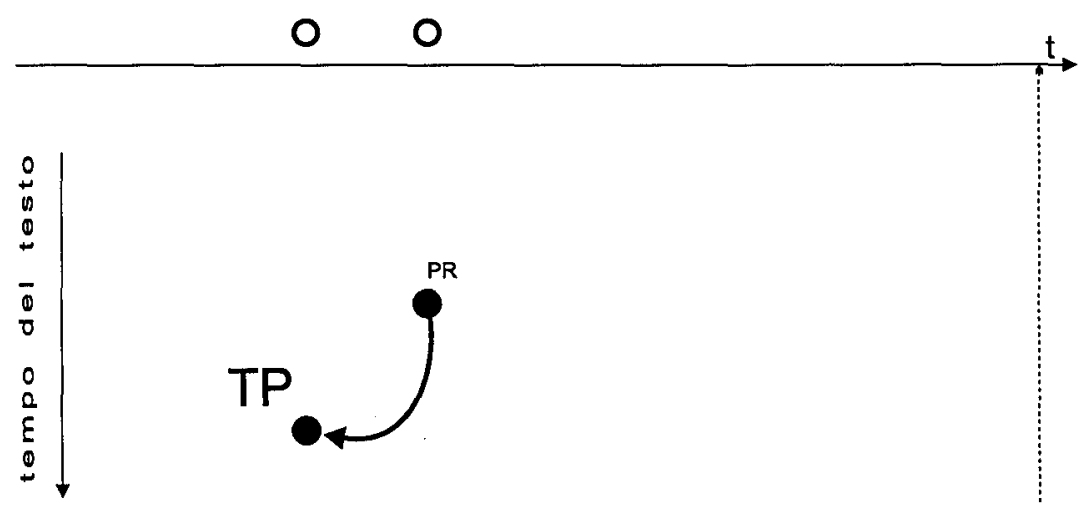

graf. 1: flash-back

\section{Preludio}

L'uso che costituisce il vero tema della presente esposizione è però un altro. Si tratta di occorrenze del TP all'inizio del testo o di una sua parte relativamente indipendente - ad es. come nel soprammenzionato inizio del L.5. Abbiamo già ricordato i due punti temporali intrinsechi nel TP: il punto di riferimento (per lo più conosciuto dal contesto precedente) e la posizione temporale dell' azione designata. Se il TP appare nel testo in un momento in cui il punto di riferimento non è ancora conosciuto, significa che viene presentata per prima l' azione a sinistra, mentre l' azione che contiene anche il punto di riferimento, necessariamente successivo nel tempo, rimane, annunciata com'è cataforicamente, ancora sconosciuta e verrà offerta solo dal co-testo successivo. In 
questo modo nell'ascoltatore/lettore si crea una specie di tensione, curiosità, orientamento in avanti. Si veda l'illustrazione nel grafico No 2.

tempo del mondo testuale

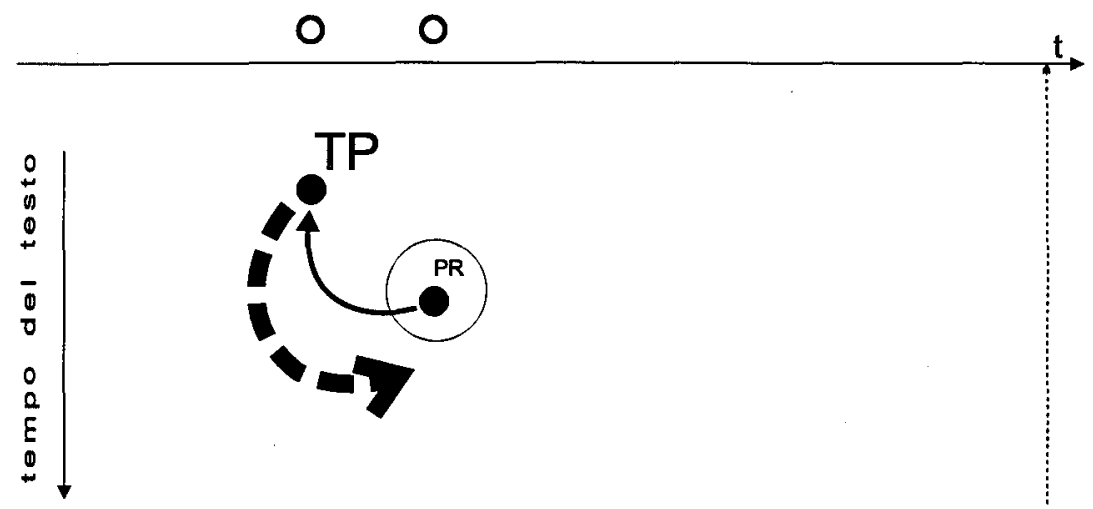

graf. 2: preludio

Con tale impiego non naturale, cataforico, è come se il TP togliesse alla propria azione una parte della rilevanza spostandola generosamente alla preannunciata azione focale, designata da un paradigma narrativo neutrale (ad es. dal Passato remoto (PR) o dal Passato prossimo (PP)). L'opposizione tra la presentazione di due azioni successive all'ordine naturale $(\mathrm{PR}+\mathrm{PR})$ e quella al preludio $(\mathrm{TP}+\mathrm{PR})$ potrebbe essere illustrata dal grafico No 3.
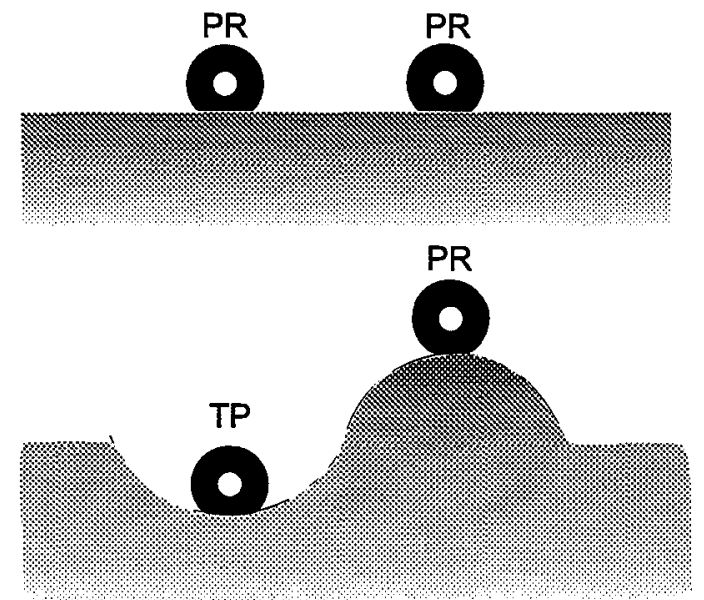

\section{graf. 3: ordine naturale e preludio}

Le azioni al TP sembrano di conseguenza relegate in secondo piano, o fuori dal fuoco narrativo, mentre, per contrasto, le azioni al PR acquistano maggior rilievo. 
Così nel capitolo II.5, dedicato all'esposizione delle passeggiate per il rigoglioso parco dell'io narrante (chiamiamolo Giorgio) con Micòl, la figlia dei proprietari, dalla narrazione delle azioni al Passato remoto (ad es. "tornò seria") Bassani passa al Trapassato per designare un'azione che è in realtà posteriore (s'era messo a piovere). L'inizio della pioggia in quel momento infatti non è presentato alla pari con le principali azioni della narrazione, bensì ha la semplice funzione - di preludio appunto di introdurre le azioni di primaria importanza (a partire da attraversammo):

Tornò seria, e poi: (...)

Un altro giorno, l'ultimo, S'ERA MESSO a piovere, e mentre gli altri riparavano nella Hütte a giocare a ramino e a ping-pong noi due, incuranti di inzupparci attraversammo correndo mezzo parco per andare a rifugiarci nella rimessa (GFC 91)

Similmente nel capitolo IV.8, dove le singole azioni della narrazione vengono segnalate dai PR (ad es. a p. 216: "tacque", "ammise", "soggiunse"), a p. 217 Bassani impiega una serie di Trapassati per aumentare l'effetto dell'azione espressa alla fine con il PR ("chiese"): il sorprendente suggerimento dell'amico Malnate di entrare in un bordello:

Una notte dopo ferragosto, tardi, CI ERAVAMO FERMATI a bere del vino in una fiaschetteria di via Gorgadello, di fianco al Duomo, a pochi passi di distanza da quello che fino a un anno e mezzo prima era stato l'ambulatorio medico del dottor Fadigati, il noto otorinolaringoiatra. Tra un bicchiere e l'altro AVEVO RACCONTATO a Malnate la storia del dottore. (...)(AVEVO DETTQ "per amore": e Malnate non ERA RIUSCITO a risparmiarsi una risatina sarcastica, di tipo prettamente goliardico). Da Fadigati a venire a parlare dell'omossessualità in genere il passo ERA STATO breve. (...) AVEVO RAGGIUNTO (...) CI ERAVAMO ACCANITI (...) ERAVAMO SCOPPIATI in una risata (...) AVEVAMO ATTRAVERSATO (...) AVEVA ATTACCATO (...)

Declamava a mezza voce, nel tono amaro e doloroso che sempre assumeva quando aveva scelto il Lament: (...) ma qui di colpo SI ERA INTERROTTO.

"Cosa ne diresti" mi chiese, e accennava col mento alla porta di un postribolo, "se entrassimo a vedere?" (GFC 217-218)

\subsection{Diffusione del preludio}

Questo espediente retorico non è per niente limitato all'uso letterario: l'impiego del preludio si incontra infatti in svariati tipi di testi. ${ }^{1}$ Così ad es. nei compendi di opere letterarie del Dizionario Bompiani:

L'ANIMA CHE OPERA GUARIGIONI (Die Heilung durch den Geist). Sono tre saggi, pubblicati nel 1931, dello scrittore austriaco Stefan Zweig (1881-1942), su F. A. Mesmer, M. Baker Eddy, S. Freud. I saggi espongono 
ciclicamente uno stesso problema: la guarigione psichica delle malattie. L'antichità e il Medioevo già ERANQ RICORSI, sotto il velo della religione, a pratiche psicoterapeutiche: ma il primo che si occupò scientificamente del problema fu il tedesco Franz Anton Mesmer (...) (BOM A-B 188)

nella critica letteraria, rappresentata qui dall' inizio di un capitolo del libro di A. Bon Come leggere Il giardino dei Finzi-Contini di Giorgio Bassani:

\section{COMMENTO CRITICO}

Già nei giudizi più "a caldo" la critica AVEVA SOTTOLINEATO come questo "romanzo della segregazione e della morte, del quale la tomba di famiglia rimasta vuota dà sin dall'inizio la chiave, riprende, con quale ampiezza, tutti i temi che Bassani aveva sin qui trattati". Così A. Palermo osservan che (...) (Bon 51)

nei quotidiani:

Insidiava una bimba di 9 anni.

Lucca, pedofilo finisce in cella

LUCCA - AVEVA ATTIRATO l'attenzione di una bimba di nove anni offrendole caramelle. I carabinieri di Altopascio, appostati nei pressi, lo hanno sorpreso mentre era in atteggiamenti inequivocabili con la piccola e lo hanno arrestato. In carcere è finito un settantenne con l'accusa di violenza sessuale su minori. (la Repubblica, 23 Sett. 1998, p.23)

1 Il preludio inoltre non è una particolarità dell'italiano, bensì è sfruttato in maggiore o minore misura anche in altre lingue. Lo illustro, per il tedesco, con il riassunto di un'opera letteraria, dove le azioni centrali espresse dal PRAESENS vengono preannunciate da predicati al PLUSQUAMPERFEKT, PERFEKT e PRAETERITUM:

- Aegidius, ein Athener aus vornehmer Familie, der bei einem Eremiten in Südfrankreich Aufnahme GEFUNDEN HATTE und nach seiner Bekehrung bald wegen seiner Wunder wirkenden Gebete berühmt GEWORDEN WAR HAT sich, da er "gotis hulde" zu verlieren FÜRCHTETE, völlig in die Wildnis ZURÜCKGEZOGEN; nachdem er lange Zeit nur von Kräutern und Wasser GELEBT HAT, schickt ihm Gott "eine herliche hindin", deren Milch ihn nun täglich stärkt. (Kindlers Literatur Lexikon - Aegidius)

Nello sloveno odierno, il preludio è praticamente sconosciuto. Lo troviamo invece nei testi del secolo scorso, quando il predpreteklik - una specie di trapassato sloveno - veniva usato ancora regolarmente. Ecco due esempi, firmati dal letterato Fran Levstik per la rivista satirica Pavliha:

V Ljubljani SMO SE BULI on dan SEŠLI, kar nas je največjih slovenskih prvakov, da bi se zopet pomirili med seboj. Najbolj je tržaški g. Cegnar sam sebe in tudi nas miril od jutra do večera in še tri dni potlej, ko smo vže bili vsi mirni. Tedaj smo izvolili g. Svetca, da je sestavil naslednjo pogodbo, katero smo vsi podpisali: (...) (Pavliha, 31. maja 1970, p. 11)

oppure in combinazione con il historični sedanjik, il "presente storico" sloveno:

Pavliha.

Te dní SEM BIL skrivaj PRIŠEL v Ljubljano, da bi na svoje oči videl, kako se delajo viharne priprave bližnjim volitvam. Ker se nikakor nijsem drznil stopiti v narodno "Slovenijo," oblečem svobodoljubno suknjo, pokrijem svobodoljubni cilinder, vzamem (...) (Pavliha, 30. junija 1870, p. 19) 
L'autrice di "Va' dove ti porta il cuore" non riusciva a mettersi in contatto e ha chiesto un controllo. Il medico legale: un malore.

Trovato morto il padre della Tamaro

Il corpo nella casa di Roma. Un'amica della scrittrice dà l'allarme

ROMA - AVEVA RILASCIATO un' intervista in mattinata, per presentare il suo ultimo libro che parla di morte e dolore. E il dolore più atroce per Susanna Tamaro, l'autrice di "Va' dove ti porta il cuore" è arrivato poche ore dopo: la morte del padre, Giovanni, settant'anni, trovato cadavere alle 7 di sera in un appartamentino di via del Lago Terrione, una strada a pochi passi da via Gregorio VII. (la Repubblica, 23 Sett. 1998, p.22)

nelle riviste:

Non ci sarà nessuno ad aspettarmi, ne sono sicura. Ho imparato a mie spese quanto sia poco gratificante il ruolo di moglie ..., AVEVA DETTO Franca Rame, sul piccolo aereo che la mattina del 10 dicembre la catapultava a Stoccolma, giusto qualche ora prima che cominciassero le celebrazioni di un Nobel diverso da tutti gli altri. (...) Ma Stoccolma non è la Milano del sindaco Albertini, dei letterati rancorosi e delle croniste del bon ton. $\mathrm{E}$ così molti giornalisti e molte tv si sono spinti fino alla gelida pista dell'aeroporto per far conoscere agli svedesi l'altra metà del Nobel. Grande da queste parti è la curiosità per Franca Rame, (...) (L'altra metà del Nobel, intervista di Chiara Valentini con Franca Rame. L'Espresso N.50, 18 Dic.1997, p. 74)

in diverse rubriche della rivista La Settimana Enigmistica:

\section{SUSPENSE!}

Il signor Brando sta raccontando ad un conoscente un fatto accaduto ad un suo lontano parente nel secolo scorso. Questi, che si chiamava Ubaldo, ERA STATO gravemente OFFESO da un certo signor Filippo, per cui lo AVEVA SFIDATO a duello. Come arma, ERA STATA SCELTA la pistola e SI ERA FISSATO il luogo dello scontro ad una distanza di circa un chilometro dalla casa di Ubaldo. La mattina del duello, Clara, la moglie di Ubaldo, stava su un balcone della casa in preda a comprensibile ansia: ella sapeva che il marito avrebbe dovuto sparare per primo, per cui, se avesse udito un secondo colpo d'arma da fuoco, avrebbe dovuto temere seriamente per la vita del suo sposo, poiché era ben noto che Filippo non falliva mai il bersaglio e, purtroppo, furono proprio due i colpi che la signora Clara sentì. Ciò nonostante, con intensa emozione ed immensa gioia, pochi minuti più tardi ella vide Ubaldo che tornava sano e salvo verso casa.

Sapreste dire come può essere spiegato questo fatto? (LSE 2836-30) 
LEGGENDO QUA E LÀ...

La statunitense Elfie Wade, un'accanita fan del celebre attore Sylvester Stallone, ERA ARRIVATA ad assillarlo in modo insostenibile, scrivendogli, in 20 mesi, più di 200 tra lettere e cartoline. Un tribunale di Los Angeles ha ora impostio alla donna di tenersi ad almeno 300 metri di distanza dall'abitazione di Stallone e di non scrivergli, e tanto meno di telefonargli, per i prossimi tre anni. (LSE 3471-4)

FORSE NON TUTTI SANNO CHE...:

In previsione delle proprie nozze con la cugina Sofia, re Luigi II di Baviera (1845-86) AVEVA FATTO costruire una carrozza che ERA VENUTA a costare una cifra astronomica: il cocchio, però, non venne mai usato, perché il sovrano, che già manifestava segni della prossima follia, ruppe il fidanzamento. (LSE 3144-19)

STRANO, MA VERO!

Per elevare il deprimente grado di cultura dei giovani della nobiltà, l'imperatore Carlo Magno AVEVA INCARICATO Alcuino, il monaco sassone consigliere di Corte, di prendersi cura della loro istruzione. Ma quando notò con quanto poco interesse venivano seguite le lezioni di quel dotto, il sovrano rafforzò l'amore degli allievi per la scienza in un modo semplicissimo: decise di assistere egli stesso alle lezioni, spesso accompagnato dalla propria moglie. (LSE 2804-39)

SE VOI FOSTE $\mathbb{L}$ GIUDICE

La Casa editrice XYZ AVEVA PRESO in affitto alcuni locali nello scantinato d'un vasto edificio e vi AVEVA DEPOSITATO libri di sua proprietà. Un giorno, per il cattivo funzionamento d'una pompa sistemata nel vano caldaia del fabbricato, si verificò l'allagamento dei locali di cui la Casa editrice era conduttrice e la maggior parte dei libri subì notevoli danni. Dopo avere invano richiesto il risarcimento al condominio, essa si decise ad intraprendere un' azione legale contro quest'ultimo. (...) (LSE 3199-16)

o nel discorso del personaggio del fumetto - imitazione della lingua parlata - nella rubrica ENIGMA POLIZIESCO ILLUSTRATO:

"Oh, ispettore Ulisse, meno male! Ho un morto a bordo! Quest'uomo ERA SALITO con una signora e mi ha chiesto di portarli in via Verdi. Là, la donna è scesa subito, dicendomi di proseguire in fretta fino allo stadio con il signore." (LSE 2615-23)

Una specie di preludio nell'effettiva comunicazione quotidiana potrebbe essere ravvisato anche nel seguente messaggio scritto su un foglio:

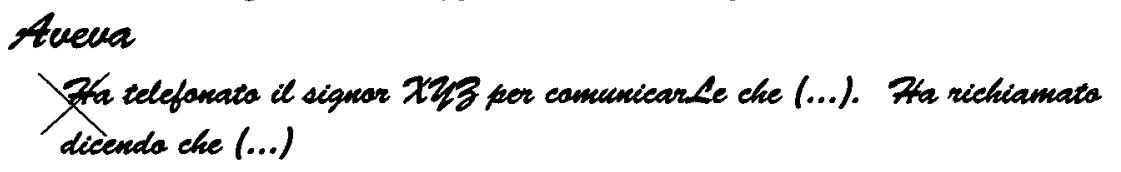


Si tratta di una mia esperienza personale di molti anni fa in una pensione di Firenze. La proprietaria aveva inizialmente scritto il primo enunciato, e dopo la seconda telefonata evidentemente non era disposta a mutilare l'informazione sull'accaduto cancellando semplicemente il primo messaggio: preferì, invece, segnalare esplicitamente il suo valore provvisorio, sostituendo il Passato prossimo con il Trapassato.

\subsection{Preludio nel procedimento storico}

In realtà il Trapassato non è l'unico paradigma in grado di svolgere la funzione di preludio. Nell'ambito del procedimento storico (cioè la narrazione del passato per mezzo di Presente, Futuro, Passato prossimo, Condizionale ecc. storici), il ruolo può essere svolto dal Passato prossimo storico. Si osservino gli esempi presi dal Dizionario Bompiani:

IL POETA E IL CONTADINO (Dichter und Bauer). Operetta in tre atti di Franz von Suppè (1819-1895), rappresentata a Vienna nel 1846. Nel piccolo albergo d'un villaggio alpestre È GIUNTO il poeta Ferdinand Rümer, che nella riposante pace del luogo spera di trovar conforto al dolore per il recente abbandono della sua amante. Egli qui si invaghisce della graziosa Lina, (...) (BOM PO-RH 181)

e da vari tipi testuali nella rivista La Settimana Enigmistica, come ad es. in una barzelletta nella rubrica RISATE A DENTI STRETTI:

Uno scozzese È appena TORNATO da un viaggio sul continente ed un amico gli chiede: (...) (LSE 3133-10)

e nelle didascalie introduttive della rubrica ENIGMA POLIZIESCO ILLUSTRATO:

Un informatore HA AVVERTITO l'ispettore Martin che in un palazzo sta per avvenire un furto. Il funzionario si apposta nei pressi. (LSE 2830-23)

1 Sul grande yacht Domedì È AVVENUTO un fattaccio incredibile: assalto di pirati, con un morto. Arriva un battello della Polizia con il commissario Parix. (LSE 3258-27)

1 Taddeo, un criminale autore di una clamorosa rapina, È EVASO dal carcere.

La fuga viene scoperta alle 5 di mattina da una guardia, che dà l'allarme.

(LSE 2829-23)

\subsection{Preludio a distanza}

Torniamo a questo punto al Trapassato - apparentemente immotivato - era accaduto all' inizio del I.5 del GFC, proposto in apertura della nostra discussione. A differenza dei casi appena presentati, dal co-testo linguistico, precedente o successivo, non si riusciva a ricavare il cruciale punto di riferimento. Il predicato in questione si riferisce all'incontro di Giorgio, ancora ragazzo, con Micòl Finzi-Contini davanti al 
muro del parco che circondava la loro sontuosa dimora, evento esposto poi per esteso alla fine di questo capitolo e in quello successivo (I.6).

Analisi approfondite dell'intero testo hanno individuato altri tre inspiegabili usi del Trapassato. Il.primo nel I.3, dove il TP è usato dopo il PR, sebbene l'azione denotata dal TP sia posteriore:

I.3

Nel 1914, quando il piccolo Guido morì, il professor Ermanno aveva quarantanove anni, la signora Olga ventiquattro. (...)

Alberto ERA NATO nel '15, Micòl nel '16: all'incirca miei coetanei. (GFC 19-20)

nel I.4., dove al Trapassato iniziale seguono precisazioni all'IMPERFETTO che si protraggono fino alla fine del capitolo, ma che non contengono il suo punto di riferimento:

I.4

Per quanto concerne me personalmente, nei miei rapporti con Alberto e Micòl c'ERA sempre STATO qualcosa di più intimo. Le occhiate d'intesa, i cenni confidenziali che fratello e sorella mi indirizzavano ogni qualvolta ci incontravamo nei pressi del Guarrini, non alludevano che a questo, lo sapevo bene, riguardante noi e soltanto noi. (...) E mi sorridevano, e mi ammiccavano, ambedue curiosamente invitanti: specie Micòl. (25-31)

e nel I.6, che dopo un breve intermezzo commentativo riprende l'esposizione - al PR dell'episodio sotto il muro di cinta. Con l'enunciato finale al Trapassato Bassani chiude il racconto dell'accaduto, il capitolo e al contempo anche la prima parte del romanzo:

\section{I.6}

Quanti anni sono passati da quel remoto pomeriggio di giugno?

(...)

E il suo ultimo sguardo, prima che scomparisse di là dal muro (uno sguardo accompagnato da un ammicco sorridente, proprio come quando, al Tempio, mi spiava da sotto il talèd paterno) ERA STATO per me. (40-48)

Ora, tutti i predicati al TP segnalati qui come problematici si riferiscono ai contatti tra Giorgio e Micòl (e il fratello Alberto) Finzi-Contini prima dell' avvicinamento fatale nel periodo autunno 1938 - estate 1939. È questo il periodo della storia centrale, come viene del resto esplicitamente suggerito dal primo enunciato del Prologo:

Da molti anni desideravo scrivere dei Finzi-Contini - di Micòl e di Alberto, del professor Ermanno e della signora Olga - e di quanti altri abitavano e come me frequentavano la casa di corso Ercole I d'Este, a Ferrara, poco prima che scoppiasse l'ultima guerra.

L'intera prima parte del romanzo è in realtà la preparazione del terreno per la comprensione della storia centrale, e le quattro importanti azioni al TP, senza un 
riconoscibile punto di riferimento nel co-testo immediato, lo hanno invece nel co-testo molto posteriore. Come spie particolari, preannunciano quindi le azioni nel vero fuoco narrativo, esposte poi nelle tre parti successive dell'opera.

Una presentazione grafica di questo preludio a distanza potrebbe essere fornita dal grafico No 4.

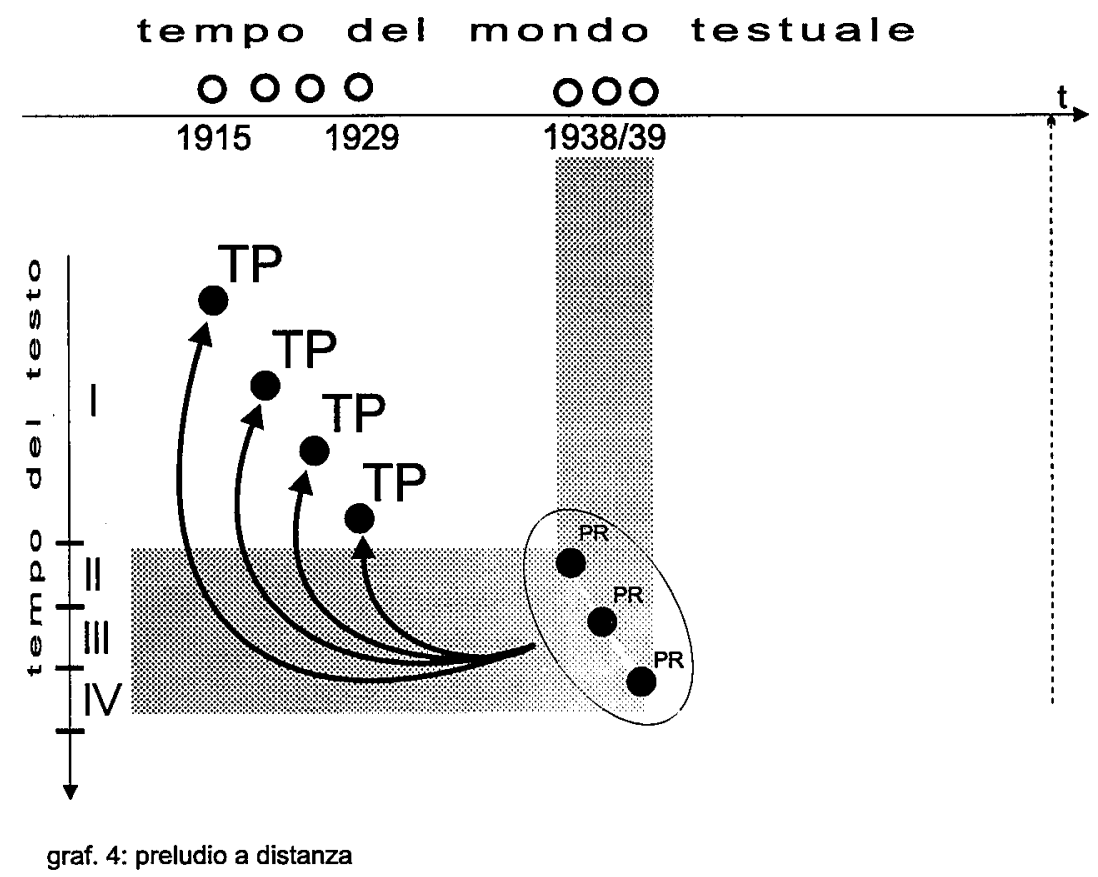

\section{Conclusione}

Abbiamo visto quindi come una particolare caratteristica formale e semantica del Trapassato prossimo (ma anche del Passato prossimo) possa essere sfruttata per impieghi retorici particolari: dall'uso primario per esprimere vari tipi di anteriorià, nonché il flash-back, attraverso il gioco di redistribuzione del peso informativo tra azioni in successione in diversi tipi di testi brevi, fino all'estensione di quest'uso a macrolivello, cioè nell' ambito globale dell'intero romanzo.

\section{Bibliografia}

ADELAR, M./LO CASCIO, V. (1986). Temporal Relation, Localization and Direction in Discourse. In: Lo Cascio, V./Vet, C. (cur.) Temporal Structure in Sentence and Discourse. 251-295. 
BAZZANELLA, C./ D. CALLERI (1991). Tense coherence and grounding in children's narrative. Text 11. 175-187.

BÄUERLE, R. (1979). Temporale Deixis, temporale Frage. Tuibingen: Niemeyer. 1937.

BASSANI, G. (1991): Il giardino dei Finzi-Contini. Milano, Arnoldo Mondadori.

BERTINETTO, P. (1986). Intrinsic and Extrinsic Temporal References: On Restricting the Notion of "Reference Time". In: Lo Cascio, V./Vet, C. (cur.) Temporal Structure in Sentence and Discourse. 49-77.

BERTINETTO, P. M. (1986). Tempo, aspetto e azione nel verbo italiano. Il sistema dell'indicativo. Firenze: Accademia della Crusca.

BERTINETTO, P. M. (1991). "Il verbo." In: Renzi/ Salvi (cur.), Grande grammatica di consultazione. II. Bologna: il Mulino. 113-161.

BERTINETTO, P. M./ M. SQUARTINI (1996). "La distribuzione del Perfetto Semplice e del Perfetto Composto nelle diverse varietà di italiano." Romance Philology XLIX/4, 384-419.

BINNICK, R. (1991). Tense and the Verb. (A Guide to Tense and Aspect.) New York/ Oxford: Oxford University Press.

BON, A. (1979). Come leggere "Il giardino dei Finzi-Contini". Milano: Mursia.

BRONZWAER, W. J. M. (1970). Tense in the Novel. (An Investigation of Some Potentialities of Linguistic Criticism.) Groningen: Wolters-Noordhoff.

CHATMAN, S. (1974). Towards a Theory of Narrative. New Literary History 6. 295318.

COMRIE, B. (1986). Tense and Time Reference: From Meaning to Interpretation in the Chronological Structure of a Text. Journal of Literary Semantics 15. 12-22.

COOPER, R. (1986). Tense and Discourse Location in Situation Semantics. Linguistics and Philosophy 9. 17-36.

CULLER, J. (1993). Fabula and Sjuzhet in the Analysis of Narrative. Poetics Today. Vol. 1(3). 27-37.

DAHL, Ö. (1985). Tense and Aspect Systems. Oxford: Blackwell.

DECLERCK, R. (1991). Tense in English. (Its structure and its use in discourse.) London and New York: Routledge.

DINSMORE, J. (1981). Tense choice and time specification in English. Linguistics 19. 475-494.

Dizionario Bompiani delle Opere e dei Personaggi. I, VII. Milano: Bompiani, 1983.

DOWTY, D. R. (1986). The Effects of Aspectual Class on the Temporal Structure of Discourse: Semantics or Pragmatics? Linguistics and Philosophy 9. 37-61.

DRY, H. (1981). Sentence Aspect and the Movement of Narrative Time. Text 1. 233240.

DRY, H. (1983). The Movement of Narrative Time. Journal of Literary Semantics 12. 19-53.

ENKVIST, N. (1981). Experiential iconicism in text strategy. Text 1. 77-111.

FLEISCHMAN, S. (1990). Tense and Narrativity. London: Routledge, 1990.

FLEISCHMAN, S. (1985). Discourse functions of tense-aspect oppositions in narrative: toward a theory of grounding. Linguistics 23. 851-882. 
FLUDERNIK, M. (1996). "Linguistics and literature: Prospects and horizons in the study of prose. Journal of Pragmatics 26. 583-611.

GENETTE, G. (1994). Die Erzählung. München: Wilhelm Fink.

GREWENDORF, G. (1982). Deixis und Anaphorik im deutschen Tempus. Papiere zur Linguistik. Nr. 26 (Heft 1/82). 47-83.

HINRICHS, E. (1986). Temporal Anaphora in Discourses of English. Lingustics and Philosophy 9. 63-82

HOPPER, P. (1979). Aspect and Foregrounding in Discourse. In: Talmy Givòn (cur.). Discourse and Syntax, Syntax and Semantics. Vol. 12. New York: Academic Press. 213-241.

HORNSTEIN, N. (1977). Towards a Theory of Tense. Linguistic Inquiry 8. 521-557.

Kindlers Literatur Lexikon. I. Zürich: Kindler, 1965.

KLEIN, W. (1977). Die Wissenschaft der Interpretation. In: Klein, W. (cur.) Methoden der Textanalyse. Heidelberg: Quelle \& Meyer. 1-23.

LEECH, G. N./ M. H. SHORT (1981). Style in fiction. London/ New York: Longman.

LO CASCIO, V. (1986). Temporal Deixis and Anaphor in Sentence and Text: Finding a Reference Time. In: Lo Cascio, V.Net, C. (cur.) Temporal Structure in Sentence and Discourse. 191-227.

LODGE, D. (1992). The art of fiction. London: Penguin.

MIKLIČ, T. (1991a). "Forme verbali italiane: come vengono presentate dalle grammatiche e come funzionano nei testi." Scuola Nostra 23, 87-103.

MIKLIČ, T. (1991b). "La forma verbale e la sua funzione nel testo: servigi testuali del trapassato del congiuntivo." In: Giannelli et al. (cur.), Tra Rinascimento e strutture attuali. Saggi di linguistica italiana. I. Torino: Rosenberg \& Sellier, 319-330.

MIKLIČ, T. (1991c). "Presenza e valori del passato remoto in riassunti di opere letterarie." Linguistica XXXI, 249-258.

MIKLIČ, T. (1997). "Segnalazione della temporalità nel testo: che cosa aiuta il ricevente a collocare le azioni sull'asse temporale." In: Agostiniani et al.(cur.), Atti del Terzo Convegno della Società Internazionale di Linguistica e Filologia Italiana. Napoli: Edizioni Scientifiche Italiane, 477-505.

MIKLIČ, T. (1998). "La distribuzione dei paradigmi verbali nei compendi di opere letterarie (italiano e tedesco a confronto.)" In: P. Cordin et al. (cur.), Parallela 6: Italiano e tedesco in contatto e a confronto. Trento: Università degli Studi, 451467.

NERBONNE, J. A. (1986). Reference Time and Time in Narration. Linguistics and Philosophy 9. 83-95.

PARTEE, B. (1973), Some Structural Analogies between Tenses and Pronouns. Journal of Philosophy 70. 601-609.

PARTEE, B. (1984). Nominal and Temporal Anaphora. Linguistics and Philosophy 7. 243-286.

PICHIASSI, M./ G. ZAGANELLI (1992). Contesti italiani: materiali per la didattica dell'italiano L2. Perugia: Guerra.

REINGART, T. (1984). Principles of Gestalt Perception in the Temporal Organization of Narrative Texts. Linguistics 22.779-809.

RICHARDS, B. (1982). Tense, Aspect and Time Adverbials. Linguistics and Pholosophy 5. 59-107. 
RICOEUR, P. (1980). Narrative Time. Critical Inquiry. 169-190.

SCHIFFRIN, D. (1981). Tense variation in narrative. Language 57(1). 45-62.

SCHOPF, A. (1984). Das Verzeitungssystem des Englischen und seine Textfunktion. Tübingen: Niemeyer.

SCHOPF, A. (1987). Essays on Tensing in English. I. Tübingen: Niemeyer.

SCHOPF, A. (1989). Essays on Tensing in English. II. Tübingen: Niemeyer.

SMITH, C. (1978). The Syntax and Interpretation of Temporal Expressions in English. Linguistics and Pholosophy 2. 43-100.

SMITH, C. (1980). Temporal Structures in Discourse. In: Christian Rohrer (cur.). Time, Tense and Quantifiers. Proceedings of the Stuttgart Conference on the Logic of Tense and Quantification. Tübingen: Niemeyer, 355-374.

SMITH, C. (1981). Semantic and Syntactic constraints on Temporal interpretation. Syntax and Semantics. Vol. 14. New York: Academic Press. 213-237.

SMITH, C. (1986). A Speaker-Based Approach to Aspect. Linguistics and Philosophy 9. 97-115.

STERNBERG, M. (1990). Telling in Time (I): Chronology and Narrative. Poetics Today 11(4). 901-948.

STERNBERG, M. (1992). Telling in Time (II): Chronology, Teleology, Narrativity. Poetics Today 13(3). 463-541.

TYLER, A. (1992). Discourse structure and specification of relationships: A cross-linguistic analysis. Text 12. 1-18.

VLACH, F. (1993). Temporal Adverbials, Tenses and the Perfect. Linguistics and Philosophy16. 231-283.

WEINRICH, H. (1964). Tempus: Besprochene und erzählte Welt. Stuttgart: Kohlhammer.

WOLFRAM, W./HATFIELD, D. (1986). Interlaguage fads and linguistic reality: The case of tense marking. In: D. Tannen - J. E. Alatis (cur.), Language and Linguistics: The Interdependence of Theory, Data, and Application

Povzetek

KATAFORIČNA RABA ITALIJANSKE GLAGOLSKE OBLIKE TRAPASSATO DELL'INDICATIVO KOT

NAČIN RELIEFIRANJA DEJANJ

Prispevek obravnava rabo italijanske glagolske paradigme Trapassato (TP) na samem začetku besedila oz. nekega njegovega relativno zaključenega dela. Pri TP gre v bistvu za tipično anaforično obliko, saj v svojem osnovnem pomenu predpostavlja premik $z$ dane referenčne točke nazaj. V zavest prikliče dve časovni poziciji: referenčno točko (praviloma znano iz predhodnega besedila) in preddobno dejanje samo. Če se TP v besedilu pojavi takrat, ko centralna točka še ni znana, pomeni da je najprej predstavljeno dejanje na skrajni levi, pri čemer ostaja časovno poznejša referenčna točka - $s$ kataforo sicer napovedana - še neznana in bo v besedilu šele sledila. Tako je ustvarjena napetost, radovednost, usmerjenost naprej. Ob tej nenaravni, kataforični rabi TP svojemu dejanju odvzame del pomembnosti in jo prenese na "napovedano" centralno dejanje, izraženo $z$ nevtralno pripovedno paradigmo (npr. s PASSATOM REMOTOM (PR)). Na ta način so dejanja $v$ TP potlačena $v$ drugi plan, dejanja v npr. PR pa dvignjena v prvi, oz. prva so izrinjena iz pripovednega gorišča, ki ga napovedujejo. Zgledi segajo od umetniških besedil prek literarne kritike in časopisnih člankov do uporabnih privatnih sporočil. 